Interview

\title{
„Wir haben eine besondere Verantwortung den Kindern gegenüber“
}

Kinderradiologin aus Leidenschaft: PD Dr. Friederike Körber gibt im Interview Einblicke in ihren Beruf und erklärt, was die Arbeit mit den jüngsten unter den Patienten so besonders macht. Sie leitet den Schwerpunkt Kinderradiologie an der Uniklinik Köln, ist Vorstandsmitglied der Deutschen Röntgengesellschaft sowie Wissenschaftliche Leiterin der Jahrestagung der Gesellschaft für Pädiatrische Radiologie (GPR), die vom 20. bis 23. September 2017 im Rahmen des Kongresses für Kinder- und Jugendmedizin in Köln stattgefunden hat.

DRG: Kinderradiologie ist ein eigener Schwerpunkt innerhalb der Radiologie. Was macht den Beruf so besonders?

PD Dr. Friederike Körber: Kinderradiologinnen und Kinderradiologen sind nicht einfach Menschen, die besonders gut mit Kindern umgehen können, sondern sie brauchen eine ganz spezielle Expertise. Das beginnt schon beim Patientenspektrum: Wir untersuchen Kinder ab dem Neugeborenenalter bis hin zu jungen Erwachsenen. In der Regel ist es so, dass ein Jugendlicher von Kinderradiologen betreut wird, solange er noch bei den Pädiatern, also Kinderärzten, in Behandlung ist. Dazu kommen ganz kleine Patienten, die schon zur Halbzeit der Schwangerschaft geboren werden (sogenannte Extrem-Frühgeborene) und im MRT auch Kinder vor der Geburt. Wir müssen eine große Vielfalt an angeborenen und erworbenen Krankheiten kennen und neben einem breiten klinischen Wissensspektrum natürlich auch technische Kenntnisse haben. Denn es gilt, die radiologische Technik auf die Kleinen und Großen anzupassen. Außerdem ist die besondere Bedeutung des Strahlenschutzes zu bedenken: Kinder und Jugendliche sind besonders strahlenempfindlich, so dass die Dosis immer im einzelnen Fall zu optimieren ist. Bei Kindern will außerdem vieles besser überlegt und begründet sein. Nehmen wir die Ultraschalluntersuchung: Natürlich ist die nicht schlimm, aber selbst der Ultraschall ist eine Untersuchung, die manchen Kleinkindern eventuell Angst macht. Wenn dann noch die großen Geräte wie der Computertomograf oder der Magnetresonanztomograf ins Spiel kommen, braucht es Empathie gegenüber den Kindern, aber auch einen guten Kontakt zu den Eltern, die ihre Ängste und Sorgen auf die Kinder übertragen können.

\section{Also werden von Kinderradiologen, im Vergleich zu anderen Radiologen, auch spezifische Psychologiekenntnisse erwartet?}

Körber: Genau. Ich kann ein Kind nicht einfach in den CT legen, ohne die Untersuchung vorher genau zu erklären. Wir sind aber keine „Wohlfühlmedizin“, sondern eine hochspezialisierte Disziplin, die viel Einfühlungsvermögen erfordert. Die Herausforderung: Wir benötigen mehr Zeit für unsere Untersuchungen und Diagnosen. Manchmal dauert die Vorbereitung, damit ein Kind eine Untersuchung überhaupt toleriert, mindestens genauso lange wie die Untersuchung und Befundung selbst. Aber das ist das Besondere an unserem Beruf: Man muss eine umfassende medizinische und technische Expertise mitbringen, und man ist dabei trotzdem sehr nah am Menschen dran. Das kommt in der Erwachsenenradiologie manchmal zu kurz. Aber in der Kinderradiologie haben wir gar keine Wahl: Wenn wir uns nicht komplett auf die Patienten und ihre Angehörigen einlassen, funktioniert die Untersuchung erst gar nicht!

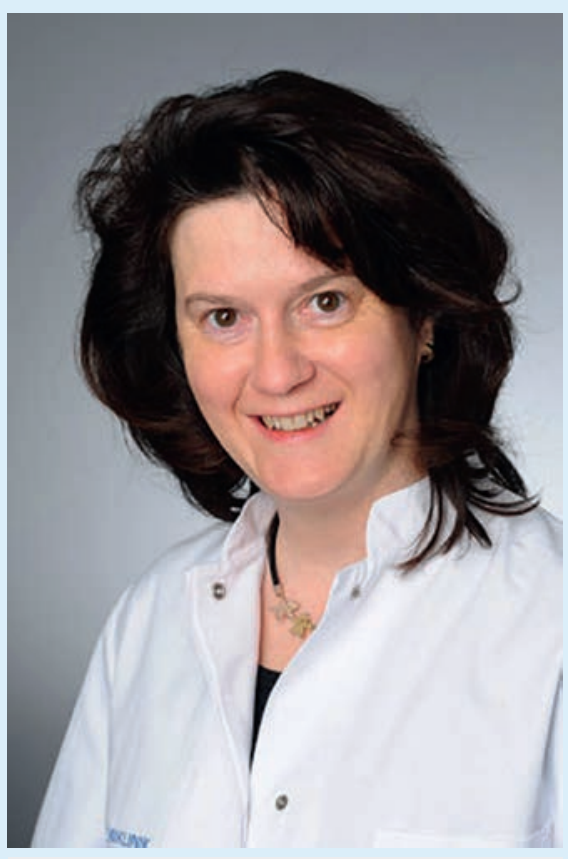

PD Dr. Friederike Körber

Müssen auch die Eltern besonders betreut werden, wenn deren Kinder untersucht werden?

Körber: Absolut. Das ist für Kollegen zu Beginn der Ausbildung häufig schwierig. Wir müssen den Eltern gegenüber vertreten, was wir tun. Eltern wollen verständlicherweise alles wissen, was die Untersuchung und vor allem die Diagnose ihres Kindes betrifft. In der Erwachsenenradiologie untersucht und diagnostiziert man als Radiologe und überlässt die Mitteilung in der Regel dem Stationsarzt. Das ist bei uns oft so nicht möglich. Das heißt, wir müssen gute Gesprächsführung beherrschen und genügend über das entsprechende Krankheitsbild wissen, damit wir auch die Folgen der Diagnose erläutern können. 
Wie sieht es mit der bundesweiten Versorgung aus - arbeiten die meisten Kinderradiologen in Praxen oder in Kliniken?

Körber: Eindeutig in Kliniken. Viele große Kinderkliniken sind in Deutschland mit kinderradiologischen Abteilungen ausgestattet. Nach meinem Kenntnisstand gibt es nur wenige niedergelassene hauptamtliche Kinderradiologen in Deutschland. Für eine Weiterbildung im Schwerpunkt Kinderradiologie ist allerdings die bundesweit recht geringe Anzahl von ausbildungsberechtigten Kinderradiologen oft ein Problem.

Müssen denn auch die Medizinisch-technischen Radiologieassistenten (MTRA) eine besondere Ausbildung für die Arbeit an Kindern mitbringen?

Körber: Es gibt zwar keine spezielle Kinderradiologieausbildung für MTRA, aber die MTRA müssen schon besondere Kenntnisse und viel Einfühlungsvermögen haben. Dafür gibt es eine intensive interne Einarbeitung zu Einstelltechniken und den Umgang mit Kindern sowie den besonderen Strahlenschutz.
Köln ist kinderradiologisch besonders gut ausgestattet: nämlich mit gleich zwei Kinderradiologien.

Körber: Das ist korrekt. Zum einen die Kinderradiologie in der Städtischen Klinik Amsterdamer Straße mit Chefarzt Dr. Martin Stenzel und die universitäre Abteilung für Kinderradiologie an der Uniklinik Köln.

Wie wichtig ist die Zusammenarbeit mit Kollegen anderer Fachrichtungen für Ihre Arbeit?

Körber: Im Alltag habe ich mindestens genauso viel mit den Kindermedizinern wie mit den Radiologen zu tun. Das heißt, ich bespreche die unterschiedlichen Untersuchungsmöglichkeiten mit den Kinderärzten und wir entscheiden gemeinsam den sinnvollsten diagnostischen Weg individuell für den jeweiligen Patienten. Wir besprechen die rechtfertigende Indikation beim Einsatz von Röntgenstrahlen und bei CT und MRT auch die Details für den Ablauf der jeweiligen Untersuchung. Die erhobenen Befunde werden dann mit den Klinikern in gemeinsamen Besprechungen diskutiert. Häufig, vor allem bei Ultraschall- und Röntgenuntersuchungen, sind wir Kinderradiologen diejenigen, die den Eltern den Befund primär erklären.
Die Kölner Uniklinik hat übrigens die erste zertifizierte Kinderonkologie in Deutschland. Um diese zu etablieren, ist eine gewisse Anzahl an Kinderradiologen und Tumorboards nötig. Ein weiterer Schwerpunkt in Köln ist die Knochenbildgebung, denn wir haben ein Subzentrum für seltene Skeletterkrankungen bei Kindern. Andere Kliniken aus ganz Deutschland nutzen unsere Kompetenz und Ausstattung und schicken uns regelmäßig Fragen und Bilder. Eines der Hauptthemen der GPR-Jahrestagung 2017 war chronisch kranke Kinder. Das betrifft die Kinderradiologie ganz besonders in Bezug auf die „Osteogenesis imperfecta“. Wir betreuen etwa 250 Patienten. Inzwischen sind wir in Deutschland das Hauptzentrum für die Glasknochenkrankheit. Die Patienten bekommen eigentlich einmal im Jahr eine Röntgenuntersuchung der Wirbelsäule. In Köln ersetzen wir diese Kontrolluntersuchungen mittlerweile durch die sehr fortgeschrittene DXAMethode (Dual-Röntgen-Absorptiometrie; englisch: Dual-energy X-ray absorptiometry). Das senkt die Strahlendosis enorm. Dies ist allerdings eine Sondergenehmigung, die nur für diese speziellen Fälle gilt und nicht auf andere Bereiche übertragbar ist.

\section{Vielen Dank!}

